\title{
Straylight Measurements in Two Different Apodized Diffractive Multifocal Intraocular Lenses
}

Ruth Lapid-Gortzak, MD, PhD; Grzegorz Labuz, MSc; Ivanka J. van der Meulen, MD, PhD; Jan Willem van der Linden, BOpt; M. P. Mourits, MD, PhD; Tom J. van den Berg, PhD

\section{ABSTRACT}

PURPOSE: To evaluate differences in straylight between eyes implanted with a hydrophilic multifocal IOL (Seelens MF; Hanita Lenses, Hanita, Israel) and a hydrophobic multifocal IOL (SN6AD1; Alcon Laboratories, Inc., Fort Worth, TX).

METHODS: In a prospective cohort study, routinely obtained straylight measurements (C-Quant; Oculus Optikgeräte, Wetzlar, Germany) 3 months after standard phacoemulsification for either cataract or refractive lens procedures were compared. Patients were implanted with either the SeeLens MF IOL or the SN6AD1 IOL. Postoperative straylight values, visual acuity, and refractive outcomes were compared.

RESULTS: The SeeLens MF IOL was implanted in 84 eyes and the SN6AD1 IOL in 79 eyes. The difference in straylight was $0.08(P=.01)$, with the SeeLens MF IOL having less straylight. Postoperative CDVA was logMAR $-0.03 \pm 0.06$ in the SeeLens MF group, and logMAR $-0.02 \pm 0.08$ in the SN6AD1 group. Mean postoperative refraction was $+0.01 \pm 0.43$ and $+0.06 \pm 0.35$ $D$, respectively.

CONCLUSIONS: The Seelens MF IOL showed a straylight of log(s) 0.08 lower than the SN6AD1 IOL. In terms of spherical equivalent and visual acuity the lenses performed equally. More study will aid in understanding the causes and clinical impact of this difference.

[J Refract Surg. 2015;31(11):746-751.]

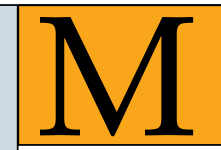

ultifocal intraocular lenses (IOLs) were introduced in cataract surgery to address the problem of loss of accommodation following lensectomy and implantation of a monofocal IOL. There have been different multifocal designs, including diffractive bifocal, ${ }^{1}$ apodized diffractive, ${ }^{2}$ trifocal, refractive, zonal refractive, ${ }^{1}$ rotational asymmetric refractive, ${ }^{3}$ and accommodating IOLs in which the optic or optics need to move to achieve accommodation. ${ }^{4}$

Bifocal diffractive apodized IOLs have a circular pattern on the optic surface, which allows for diffraction of incoming light into two main distinct foci. One focus is for distance vision and the secondary focus is for near vision. The diffraction patterns distribute the light to both foci with loss of energy; approximately $18 \%$ of light is directed outside the far and near focal points. ${ }^{2}$ As a result of multiple foci occurring in the eyeball at the same time and interfering with one another, the contrast sensitivity for both far and near foci decreases. ${ }^{5}$ In apodized diffractive IOLs, the far focus is dominant and receives more light, whereas the near focus receives less light. ${ }^{2}$ The patterns of the apodization can differ and not only allow changes in depth of focus with relation to the intensity of light distributed between the far and the near foci, but also changes in induced halos, contrast, and quality of vision with relation to the pupil size as a function of light intensity as part of the accommodative triad response. ${ }^{6,7}$

From the Department of Ophthalmology, Academic Medical Center, University of Amsterdam, the Netherlands (RL-G, IJM, MPM); Retina Total Eye Care, Driebergen, the Netherlands (RL-G, IJM, JWL); Rotterdam Ophthalmic Institute, the Netherlands (GL); and Netherlands Institute for Neurosciences, Royal Dutch Academy of Arts and Sciences, Amsterdam, the Netherlands (TJB).

Submitted: May 3, 2015; Accepted: August 18, 2015

The Royal Dutch Academy of Arts and Sciences has a proprietary interest in the C-Quant Straylight meter. Dr. Lapid-Gortzak has received speaker fees from Alcon Laboratories, Inc., and Hanita Lenses, is a clinical investigator for Alcon Laboratories, Inc., and Orca Surgical, and is a consultant for Alcon Laboratories, Inc., Hanita Lenses, Orca Surgical, and Sanoculis. The remaining authors have no financial or proprietary interest in the materials presented herein.

Correspondence: Ruth Lapid-Gortzak, MD, PhD, Department of Ophthalmology, Academic Medical Center, Meibergdreef 9, 1100 AZ Amsterdam, the Netherlands.E-mail: r.lapid@amc.nl

doi: 10.3928/1081597X-20151021-03 
Straylight is a parameter of quality of vision, and is by definition glare disability. The light does not come to a focus on the retina because of imperfections in the optical system, but is forwardly scattered in the eye and veils vision. Straylight is known to increase with the development of cataract. ${ }^{8}$ Corneal and vitreous turbidity may also increase straylight. ${ }^{9}$ Elements in the capsular bag, such as the rhexis edge, posterior capsular opacification, and edges of YAG capsulotomies contribute to straylight after surgery. ${ }^{10,11}$ Van der Meulen et al. showed that straylight decreases after cataract surgery and that preoperative straylight levels increase the predictability of the visual outcome of cataract surgery when used with visual acuity measurements. ${ }^{12}$ Straylight improves significantly in many patients after phacoemulsification in eyes with good preoperative visual acuity. ${ }^{13}$ However, the contribution of the IOL type to postoperative straylight has not yet been elucidated.

The effect of IOLs on straylight has already been studied by many authors, but the literature appears to be inconsistent. Dick et al. ${ }^{14}$ showed that there is no difference between monofocal and multifocal IOLs in terms of straylight. This is in line with findings from Hofmann et al., ${ }^{15}$ Cerviño et al., ${ }^{16}$ and Wilkins et al. ${ }^{17}$ However, de Vries et al. ${ }^{18}$ found increased straylight of $\log (\mathrm{s}) 0.078$ in eyes implanted with the SN6AD3 (Alcon Laboratories, Inc., Fort Worth, TX) and Peng et al. ${ }^{19}$ found an increase of straylight in eyes implanted with an apodized diffractive IOL. Ehmer et al. ${ }^{20}$ found increased straylight in diffractive IOLs compared to refractive IOLs. The comparison of multifocal apodized diffractive IOLs from the same manufacturer, differing only in its effect on spherical aberration, have shown no difference in straylight. ${ }^{21}$ Most of these studies were done with small cohorts, and have shown different results in terms of straylight when using different types of lenses. As a result, the effect of multifocality on straylight is unclear.

In this study, two types of optics of apodized diffractive IOLs were compared: the SN6AD1 (Alcon Laboratories, Inc.) versus the SeeLens MF (Hanita Lenses, Hanita, Israel). Both are bifocal apodized diffractive IOLs, but in one of these lenses the apodization pattern was adjusted in the number, distance, and height of the diffractive rings in an attempt to achieve a better differentiation of the foci, presenting allowance for intermediate vision and decreased halos and optical side effects. In this study, we compared the postoperative straylight in eyes implanted with either of these two lenses.

\section{PATIENTS AND METHODS}

Between April 2011 and May 2013 all consecutive patients older than 18 years undergoing a standard phacoemulsification for cataract or refractive lens ex- change were included. The tenets of the Declaration of Helsinki were adhered to, the study was approved by the institutional review board, and all patients provided a signed informed consent. The following patients were excluded: patients younger than 18 years, patients incapable of or not willing to consent, and patients with diabetes, cornea guttatae, glaucoma, uveitis, macular disease, previous corneal laser surgery, or other significant eye disease.

\section{SURGical TECHNIQUE}

A standard phacoemulsification procedure was performed using the infinity OZIL phacoemulsification technology (Alcon Laboratories, Inc.) with a 2.2-mm incision. After removal of the native lens, the posterior capsule was polished using the bimanual irrigation/ aspiration handpieces. The anterior capsule was polished in the area of the optic adhering under the rhexis. The lens implanted was either an SN6AD1 or a SeeLens MF. The choice of IOL resulted from shared decisionmaking between the patient and the eye surgeon.

\section{LENSES}

The SN6AD1 is an hydrophobic apodized diffractive IOL, with a 6-mm optic and 13-mm haptic diameter and straight posterior optic edge, no angulation, blue blocker material, with a yellowish tint. It can be injected through a $2.2-\mathrm{mm}$ opening into the bag via a cartridge system. The SN6AD1 has an apodized profile on the central $3.6 \mathrm{~mm}$ of the optic with 9 diffractive rings that have specific height and distance steps.

The Seelens MF is a hydrophilic one-piece IOL, with apodized diffractive optics. The optic is $6 \mathrm{~mm}$ and the haptic diameter is $13 \mathrm{~mm}$, with a $5^{\circ}$ posterior angulation of the optic to the C-loop haptics. The optic has a $360^{\circ}$ sharp edge to prevent posterior capsular opacification. The material has an ultraviolet blocking chromophore surface and is clear. The apodization pattern has a central diameter of $4 \mathrm{~mm}$ with 11 difractive rings that have had the step height and distance adjusted for minimal halos and glare perception, and maximal visual acuity at distance and near.

\section{Patient examinations}

A full preoperative ophthalmic examination was performed, including visual acuity and refraction, slitlamp and biomicroscopy examination, biometry (IOLMaster; Carl Zeiss AG, Jena, Germany), topography (Orbscan; Bausch \& Lomb, Rochester, NY), tonometry, tear film diagnostics, and standard straylight measurements (C-Quant; Oculus Optikgeräte, Wetzlar, Germany). The above was repeated at 3 months, except for the topography and biometry. 


\begin{tabular}{|c|c|c|c|}
\hline \multicolumn{4}{|c|}{$\begin{array}{c}\text { TAB } \angle E 1 \\
\text { Refractive Data }\end{array}$} \\
\hline Parameter & SeeLens MF & SN6AD1 & $P$ \\
\hline No. of eyes & 84 & 79 & - \\
\hline Preop CDVA (logMAR \pm SD, range) & $0.04+0.08(0.3$ to -0.1$)$ & $0.06+0.10(0.4$ to -0.1$)$ & .171 \\
\hline Postop CDVA (logMAR \pm SD, range) & $-0.03+0.06(0.2$ to -0.16$)$ & $-0.02+0.08(0.4$ to -0.2$)$ & .205 \\
\hline Preop refraction ( $S E \pm S D$, range), D & $+1.30+2.05(-6.625$ to +5.75$)$ & $+0.48+2.65(-10.75$ to +6.00$)$ & .027 \\
\hline Postop refraction (SE $\pm S D$, range), D & $0.01+0.43(-1.375$ to +1.25$)$ & $0.07+0.35(-0.75$ to +0.875$)$ & .383 \\
\hline
\end{tabular}

Preop = preoperative; $C D V A=$ corrected distance visual acuity; Postop = postoperative; $S D=$ standard deviation; $S E=$ spherical equivalent; $D=$ diopters The SeeLens MF IOL is manufactured by Hanita Lenses, Hanita, Israel. The SNGAD1 IOL is manufactured by Alcon Laboratories, Fort Worth, TX.

\begin{tabular}{|c|c|c|c|}
\hline \multicolumn{4}{|c|}{$\begin{array}{c}\text { TABLE } 2 \\
\text { Demographic Data }\end{array}$} \\
\hline Parameter & SeeLens MF & SN6AD1 & $P$ \\
\hline $\begin{array}{l}\text { Refractive lens } \\
\text { exchange (eyes) }\end{array}$ & 35 & 31 & - \\
\hline Cataract (eyes) & 49 & 48 & $<.752^{\mathrm{a}}$ \\
\hline Male/female & $46 \% / 54 \%$ & $41 \% / 59 \%$ & $<.61^{\mathrm{b}}$ \\
\hline Age, y & $59 \pm 9$ & $61 \pm 7$ & $<.14^{\mathrm{b}}$ \\
\hline \multicolumn{4}{|c|}{ 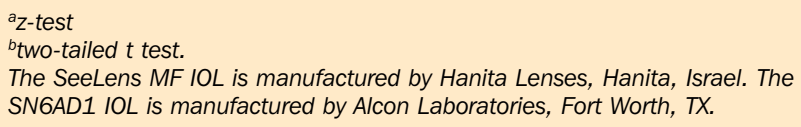 } \\
\hline
\end{tabular}

\section{STRAYLIGHT MEASUREMENTS}

Using the C-Quant straylight meter, the straylight was measured twice before and 3 months after surgery and expressed in a logarithmic scale as $\log (\mathrm{s})$. The use of the technique has been extensively described elsewhere. ${ }^{22}$ The straylight measurements were performed by the same optometrist under identical conditions. The optometrist was blinded to the fact that there was a study being done.

\section{Statistical Analysis}

Data were analyzed using statistical functions in Excel 2003 software (Microsoft Corporation, Redmond, WA). The parametric double-side $t$ test was used as applied for normally distributed data. Correlations were calculated using normal regression analysis.

\section{RESULTS}

The refractive data are summarized in Table 1. Preoperative corrected distance visual acuity was similar in both groups, as was the postoperative corrected distance visual acuity and refraction. Demographic data are summarized in Table 2.

Table 3 summarizes the straylight data. There was an overall decrease of straylight in both groups, with an improvement of $0.01 \pm 0.21 \log (\mathrm{s})(P=.61)$ in the SN6AD1 group, versus a $0.07 \pm 0.18 \log (\mathrm{s})(P<.003) \mathrm{de}-$

\begin{tabular}{|c|c|c|}
\hline \multicolumn{3}{|c|}{$\begin{array}{c}\text { TABLE } 3 \\
\text { Preoperative to Postoperative } \\
\text { Decrease in log(s) Values }\end{array}$} \\
\hline Parameter & SN6AD1 & SeeLens MF \\
\hline Preoperative log(s) & $1.19 \pm 0.21$ & $1.16 \pm 0.20$ \\
\hline Postoperative log (s) & $1.17 \pm 0.14$ & $1.10 \pm 0.19$ \\
\hline Improvement & $0.01 \pm 0.21$ & $0.08 \pm 0.18$ \\
\hline$P$ (two-tailed $t$ test) & .605 & .003 \\
\hline \multicolumn{3}{|c|}{$\begin{array}{l}\text { The SeeLens MF IOL is manufactured by Hanita Lenses, Hanita, Israel. The } \\
\text { SN6AD1 IOL is manufactured by Alcon Laboratories, Fort Worth, TX. }\end{array}$} \\
\hline
\end{tabular}

crease in the SeeLens MF group. Postoperatively there was a difference between the SN6AD1 group and the SeeLens MF group of $0.08 \log (\mathrm{s})(P=.01)$ in favor of the IOL with the adjustment in the apodization (SeeLens $\mathrm{MF}$ ). When adjusted for age, this difference remained statistically significant at $0.06 \log (\mathrm{s})(P=.03)$.

Straylight improved most in eyes that had higher preoperative levels of straylight (Figure 1). When the change in straylight was plotted against the graph showing the normal phakic norm with age (Figure 2), it is clear that straylight was reduced postoperatively with both multifocal IOLs. The improvement was greatest using the SeeLens MF, as compared to the SN6AD1.

None of the eyes had significant glistenings. At the 3-month follow-up visit, none of the eyes had posterior capsular opacification and none of the eyes had undergone a posterior capsulotomy for that reason.

\section{DISCUSSION}

Straylight decreased postoperatively in both groups. The mean difference between age-adjusted groups was $0.06 \log (\mathrm{s})(P<.03)$ in favor of the SeeLens MF IOL. A difference of $\log (\mathrm{s})$ of 0.06 can be compared to the logMAR scale on the visual acuity chart, and would in comparison be a difference of "3 letters" on the visual acuity chart. This is a small but statistically significant difference. 


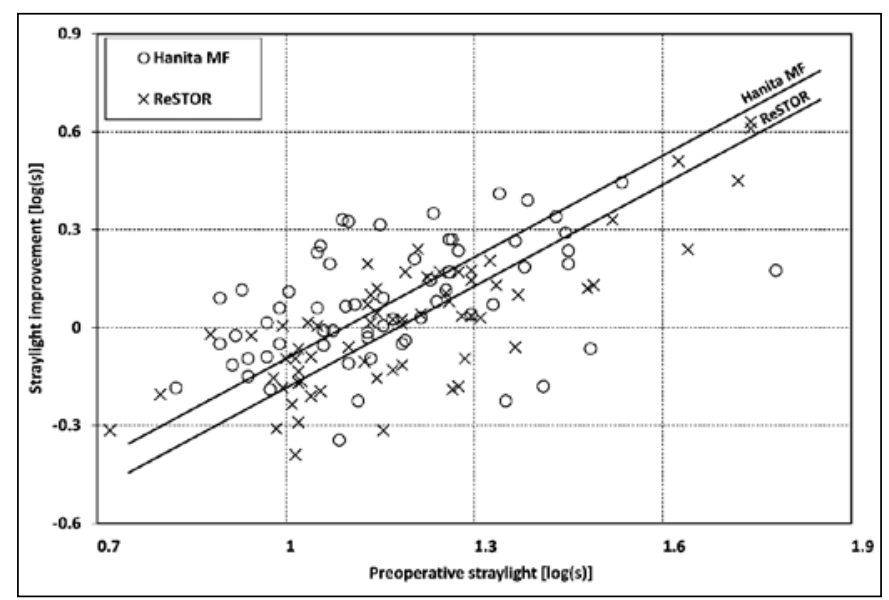

Figure 1. Straylight improved in the patient population overall. The improvement in straylight was greatest in eyes with higher preoperative straylight.

The explanation of this finding may lie in three factors. The first is the difference between the materials. Montenegro et al. ${ }^{23}$ showed that the hydrophilic acrylic IOLs induce significantly less straylight than the hydrophobic IOLs. Moreover, the hydrophobic AcrySof material (Alcon Laboratories, Inc.) has a tendency for glistenings ${ }^{24}$-incorporating small inclusions of water and calcium in the open spaced polymer structure. However, the 'defects' caused by glistenings are deemed too small to influence straylight. ${ }^{25}$ In this study, none of the IOLs showed significant glistenings in the 3 months of follow-up. As a result, glistenings do not seem to play a role in the higher straylight of the hydrophobic lens group. The second factor may be the apodization pattern, and the third factor is the manufacturing process of the hydrophilic lenses, which makes the optic surface of these hydrophilic lenses accurate. ${ }^{26}$ One may be inclined to think that the asphericity patterns of the IOLs influence straylight, but because wavefront aberration affects the peak of the point spread function only and straylight is $1^{\circ}$ or more off from peak, there is no physical overlap between the two functions, and as such different asphericity will have no effect on straylight. ${ }^{27}$

The apodization pattern is the pattern in which the steps of the diffractive rings are spaced at different distances from the center of the lens and at different height to get a better energy balance. When the SN6AD3 with the +4.00 addition for runner of the SN6AD1 was designed, the primary guiding principle was that near vision is less important than distance vision, when in dim illumination when pupils are large. Clinically, this can be understood by the vision tasks in the dark (ie, driving) where a distance dominance is important, as opposed to near tasks, which need better illumination,

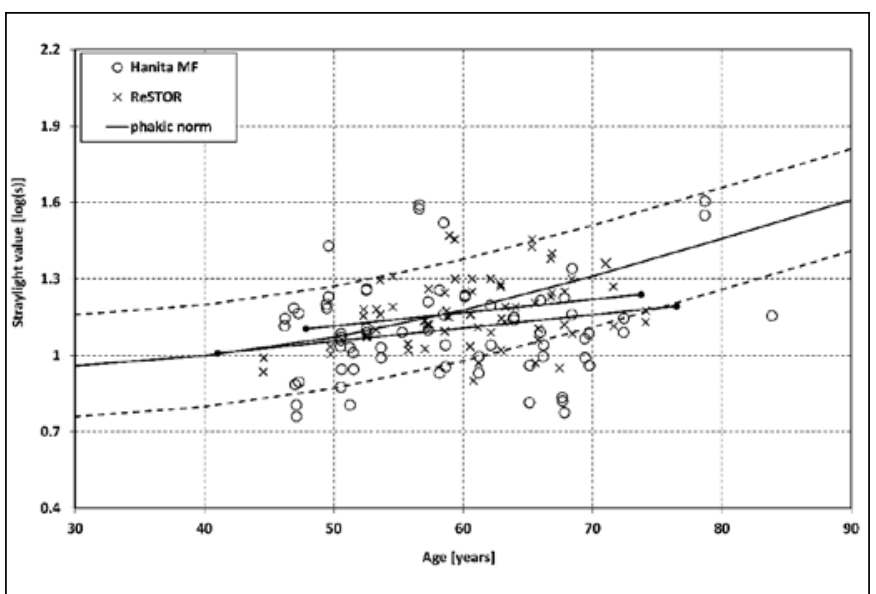

Figure 2. Straylight postoperatively in the multifocally pseudophakic eyes. The upper regression line is for the SN6AD1 (Alcon Laboratories, Inc., Fort Worth, TX) implanted eyes, and the lower regression line is for the SeeLens MF (Hanita Lenses, Hanita, Israel) implanted eyes. The latter have better straylight value than the former.

and will be accompanied by the synkinetic reflex. The second guiding principle was that glare and halo perception must be minimized under dim lighting conditions. $^{2}$

The glare and halos mostly result from perception of the second unfocused near image in dim illumination. These principles are complementary on apodized diffractive lenses because the balance in the energy of light going for the distance and near focus together with pupil dependency allow for manipulation of image quality. ${ }^{2}$

Gatinel et al. ${ }^{26}$ described the manufacturing process of the hydrophilic multifocal IOL in their study of the trifocal IOL. The hydrophilic multifocal IOLs are manufactured by a lathe-milling process, just like the monofocal IOLs, but the polishing step is omitted. High-precision lathes allow for low roughness and polishing-free IOLs. This is a well-accepted method for manufacture of hydrophilic multifocal IOLs and also the way the SeeLens MF lenses are manufactured. The hydrophobic IOL is cast-molded, as customary with acrylic IOLs. Surface roughness can be measured with atomic force microscopy and is possibly related to posterior capsular opacification. ${ }^{28}$ Interestingly, in this study ${ }^{28}$ the hydrophobic lenses had the least surface roughness and least posterior capsular opacification, but none of the IOLs measured in these studies were multifocal diffractive lenses, so empiric data on surface roughness of multifocal IOLs are lacking in the literature.

In comparison to the literature, this is the first study in which two different types of multifocal diffractive apodized IOLs were compared. De Vries et al. ${ }^{21}$ showed that there was no difference between spherical and aspheric multifocal IOLs with a similar material and 
diffractive pattern with respect to the mean straylight value. Van Gaalen et al. reported similar outcomes, but their study included only monofocal IOLs. ${ }^{29}$ Ehmer et al. ${ }^{20}$ assessed three types of multifocal IOLs: the AMO ReZoom (Abbott Medical Optics, Abbott Park, IL), the AMO ZM900 (Abbott Medical Optics), and the LS-312 (Oculentis Optikgeräte). The diffractive IOLs showed a higher postoperative straylight value than the symmetric and rotational asymmetric refractive IOLs. This study also reported the subjective photic phenomena, which correlated weakly with straylight. The significance of the findings is difficult to gauge because there were 10 eyes in each group. More often, comparisons between monofocal and multifocal lenses were made. It is clear that multifocal IOLs improve the depth of field at a cost of image quality ${ }^{30}$; however, there are still many doubts whether multifocality might be related to increase of straylight. Dick et al., ${ }^{14}$ Cerviño et al. ${ }^{16}$ and Wilkins et al. ${ }^{17}$ have all shown that there is no difference between monofocal and multifocal IOLs regarding straylight. Hofmann et al. ${ }^{15}$ and de Vries et al. ${ }^{18}$ separately reported more straylight by and average of $0.08 \log (\mathrm{s})$ in the multifocal group, which in the report by Hofmann et al. did not reach statistical significance. Peng et al. ${ }^{19}$ demonstrated that the apodized diffractive IOLs were associated with increased straylight and the increasing prevalence of photopic disturbances (glare and halo) compared to the monofocal IOLs. There is no clear explanation for the discrepancies in outcomes of straylight measurements in multifocal IOLs. Some reports state that the pupil size is possibly important. However, most reports have small sample sizes, and as such it is difficult to extrapolate the effect of multifocality on straylight. Optical bench systems will allow for more objective measurements. ${ }^{15,16}$

The contribution of the IOL material versus the apodization pattern is unclear. It is clear that apodization allows for better enhancement of distance and near foci. By adjusting the pupil dependency of the diffractive rings, even an IOL with an optic of 11 rings such as the SeeLens MF shows less straylight than an apodized diffractive IOL with 9 rings (SN6AD1). Improved apodization patterns may have a good effect on side effects such as halos and glare (disability glare), ${ }^{31}$ but more study is necessary to elucidate the exact contribution of the IOL material.

\section{AUTHOR CONTRIBUTIONS}

Study concept and design (RL-G, TJB); data collection (RL-G, MPM, $I J M, J W L)$; analysis and interpretation of data (GL, $R L-G)$; writing the manuscript ( $G L, R L-G$, JWL); critical revision of the manuscript ( $R L-G$, MPM, IJM, TJB); statistical expertise (TJB); administrative, technical, or material support (MPM, IJM, JWL); supervision (RL-G)

\section{REFERENCES}

1. Walkow T, Liekfeld A, Anders N, Pham DT, Hartmann C, Wollensak J. A prospective evaluation of a diffractive versus a refractive designed multifocal intraocular lens. Ophthalmology. 1997;104:1380-1386.

2. Davison JA, Simpson MJ. History and development of the apodized diffractive intraocular lens. J Cataract Refract Surg. 2006;32:849-858.

3. Venter JA, Pelouskova M, Collins BM, Schallhorn SC, Hannan SJ. Visual outcomes and patient satisfaction in 9366 eyes using a refractive segmented multifocal intraocular lens. J Cataract Refract Surg. 2013;39:1477-1484.

4. Cumming JS, Colvard DM, Dell SJ, et al. Clinical evaluation of the Crystalens AT-45 accommodating intraocular lens: results of the U.S. Food and Drug Administration clinical trial. J Cataract Refract Surg. 2006;32:812-825.

5. Allen ED, Burton RL, Webber SK, et al. Comparison of a diffractive bifocal and a monofocal intraocular lens.J Cataract Refract Surg. 1996;22:446-451.

6. Alfonso JF, Fernández-Vega L, Baamonde MB, Montés-Micó R. Correlation of pupil size with visual acuity and contrast sensitivity after implantation of an apodized diffractive intraocular lens. J Cataract Refract Surg. 2007;33:430-438.

7. Alba-Bueno F, Vega F, Millán MS. Halos and multifocal intraocular lenses: origin and interpretation [article in Spanish]. Arch Soc Esp Oftalmol. 2014;89:397-404.

8. de Waard PW, IJspeert JK, van den Berg TJ, de Jong PT. Intraocular light scattering in age-related cataracts. Invest Ophthalmol Vis Sci. 1992;33:618-625.

9. Mura M, Engelbrecht LA, de Smet MD, Papadaki TG, van den Berg TJ, Tan HS. Surgery for floaters. Ophthalmology. 2011;118:1894.

10. van der Meulen IJ, Engelbrecht LA, Van Riet TC, et al. Contributions of the capsulorrhexis to straylight. Arch Ophthalmol. 2009;127:1290-1295.

11. Lapid-Gortzak R, van der Meulen IJ, Nieuwendaal CP, van den Berg TJ. Alleviating debilitating photophobia and secondary exotropia caused by increased straylight by widening a small posterior capsulotomy. J Cataract Refract Surg. 2011;37:413414.

12. van der Meulen IJ, Gjertsen J, Kruijt B, et al. Straylight measurements as an indication for cataract surgery. J Cataract Refract Surg. 2012;38:840-848.

13. Lapid-Gortzak R, van der Meulen IJ, van der Linden JW, Mourits MP, van den Berg TJ. Straylight before and after phacoemulsification in eyes with preoperative corrected distance visual acuity better than $0.1 \operatorname{logMAR}$. J Cataract Refract Surg. 2014;40:748-755.

14. Dick HB, Krummenauer F, Schwenn O, Krist R, Pfeiffer N. Objective and subjective evaluation of photic phenomena after monofocal and multifocal intraocular lens implantation. Ophthalmology. 1999;106:1878-1886.

15. Hofmann T, Zuberbuhler B, Cerviño A, Montés-Micó R, Haefliger E. Retinal straylight and complaint scores 18 months after implantation of the AcrySof monofocal and ReSTOR diffractive intraocular lenses. J Refract Surg. 2009;25:485-492.

16. Cerviño A, Hosking SL, Montés-Micó R, Alió JL. Retinal straylight in patients with monofocal and multifocal intraocular lenses. J Cataract Refract Surg. 2008;34:441-446.

17. Wilkins MR, Allan BD, Rubin GS, et al. Randomized trial of multifocal intraocular lenses versus monovision after bilateral cataract surgery. Ophthalmology. 2013;120:2449-2455.

18. de Vries NE, Franssen L, Webers CA, et al. Intraocular straylight 
after implantation of the multifocal AcrySof ReSTOR SA60D3 diffractive intraocular lens. J Cataract Refract Surg. 2008;34:957-962.

19. Peng C, Zhao J, Ma L, Qu B, Sun Q, Zhang J. Optical performance after bilateral implantation of apodized aspheric diffractive multifocal intraocular lenses with +3.00 - $\mathrm{D}$ addition power. Acta Ophthalmol. 2009;90:e586-e593.

20. Ehmer A, Rabsilber TM, Mannsfeld A, Sanchez MJ, Holzer MP, Auffarth GU. Influence of different multifocal intraocular lens concepts on retinal stray light parameters [article in German]. Ophthalmologe. 2011;108:952-956.

21. de Vries NE, Webers CA, Verbakel F, et al. Visual outcome and patient satisfaction after multifocal intraocular lens implantation: aspheric versus spherical design. J Cataract Refract Surg. 2010;36:1897-1904.

22. Franssen L, Coppens JE, van den Berg TJ. Compensation comparison method for assessment of retinal straylight. Invest Ophthalmol Vis Sci. 2006;47:768-776.

23. Montenegro GA, Marvan P, Dexl A, et al. Posterior capsule opacification assessment and factors that influence visual quality after posterior capsulotomy. Am J Ophthalmol. 2010;150:248-253.

24. Werner L. Glistenings and surface light scattering in intraocular lenses. J Cataract Refract Surg. 2010;36:398-420.
25. Colin J, Orignac I. Glistenings on intraocular lenses in healthy eyes: effects and associations. J Refract Surg. 2011;27:869-875.

26. Gatinel D, Pagnoulle C, Houbrechts Y, Gobin L. Design and qualification of a diffractive trifocal optical profile for intraocular lenses. J Cataract Refract Surg. 2011;37:2060-2067.

27. Franssen L, Coppens JE, van den Berg TJ. Ocular media clarity and straylight. In: Dartt D, ed. Encyclopedia of the Eye. Boston: Elsevier; 2010:173-183.

28. Mukherjee R, Chaudhury K, Das S, Sengupta S, Biswas P. Posterior capsular opacification and intraocular lens surface microroughness characteristics: an atomic force microscopy study. Micron. 2012;43:937-947.

29. van Gaalen KW, Koopmans SA, Jansonius NM, Kooijman AC. Clinical comparison of the optical performance of aspheric and spherical intraocular lenses. J Cataract Refract Surg. 2010;36:34-43.

30. Holladay JT, Van Dijk H, Lang A, et al. Optical performance of multifocal intraocular lenses. J Cataract Refract Surg. 1990;16:413-422. Erratum in: J Cataract Surg. 1990;16:781.

31. van der Linden JW, van der Meulen IJ, Mourits MP, LapidGortzak R. Comparison of a hydrophilic and a hydrophobic apodized diffractive multifocal intraocular lens. Int Ophthalmol. 2013;33:493-500. 